\title{
Les procédures de décision en temps réel pour la prévision des crues
}

\author{
par Pierre-Alain Roche Ingénieur des Ponts et Chaussées, \\ et Jean-Philippe Torterotot Ingénieur du Génie Rural, des Eaux et des Forêts \\ CERGRENE Ecole Nationale des Ponts et Chaussées
}

Pour faire face à l'ensemble des perturbations susceptibles d'intervenir en temps réel dans la prévision des crues, on disposait jusqu'à une date récente de deux types de moyens: le traitement statistique du processus des écarts de prévision et le filtrage des paramètres des modèles (filtre de Kalman), les deux moyens exploitant la même information. Nous montrerons que, bien qu'issus de principes entièrement différents, ils se rejoignent d'une certaine manière en pratique, et que divers développements sont à attendre en ce qui les concerne.

Ces deux premiers moyens ne répondent que très partiellement aux situations réelles des systèmes de prévision, qui se caractérisent par la multiplicité des données collectées, et donc des combinaisons possibles de modélisation. L'essor des préoccupations de sécurité (gestion des "modes dégradés") nous a conduit à proposer une démarche multimodèle permettant d'analy. ser et d'exploiter les relations de cohérence à l'échelle d'un bassin pour accroitre la fiabilité. Cette démarche est présentée ici en liaison avec les deux moyens évoqués précédemment, et illustrée sur 2 cas d'application, la Vézère et la Garonne Amont.

\section{Le traitement des écarts d'un modèle}

\subsection{Rappel}

Nous considérons un modèle de prévision :

$$
y_{i+1}=f\left(x_{i}\right)+e_{i+1}
$$

où $y_{i+1}$ est la variable à prévoir, $x_{i}$ représente l'ensemble des données disponibles à la date de prévision, et $e_{i+1}$ est l'écart de prévision.

Le traitement des écarts a pour but de mettre au point une correction à apporter sur $f\left(x_{i}\right)$ au vu des observations $y_{i}$ et donc des écarts $e_{i}$.

On dispose de 2 types de résultats:
- pour une formule particulière de correction, par exemple :

$$
Y_{i+1}=f\left(x_{i}\right)+k\left(y_{i}-f\left(x_{i-1}\right)\right)
$$

la valeur optimale des paramètres de cette formule (ici $k$ ) se déduit des caractéristiques statistiques du processus $\left(e_{i}\right)$. Par exemple, ici, la valeur optimale de $k$ est égale au coefficient d'autocorrélation de rang 1 de $\left(e_{i}\right)$, sous l'hypothèse que celui-ci est stationnaire;

- pour une structure particulière de $\left(e_{i}\right)$, il existe une formule de correction optimale. Par exemple, si $\left(e_{i}\right)$ est un processus autorégressif de rang 1 ,

$$
e_{i+1}=r e_{i}+u_{i+1}
$$

avec $\left(u_{i}\right)$ bruit blanc : alors la correction (2) est optimale

Real time decision procedures relating to flood forecasting

This article describes with the help of application examples how it is possible to provide an elaborated forecast from the use of simulation models and various real-time decision procedures. Correction of forecast errors via the analysis of the error stochastic process is currently used. Model parameter filtering is used in special cases and its application field has been stated by many undertakings during these last ten years. More recently, procedures known as multimodels have been introduced. They involve either deciding to replace the current model in the case of failure or to combine the results with a weighting suited to the current state of the system at the time of forecast. These recent methods are presented here with more details. 

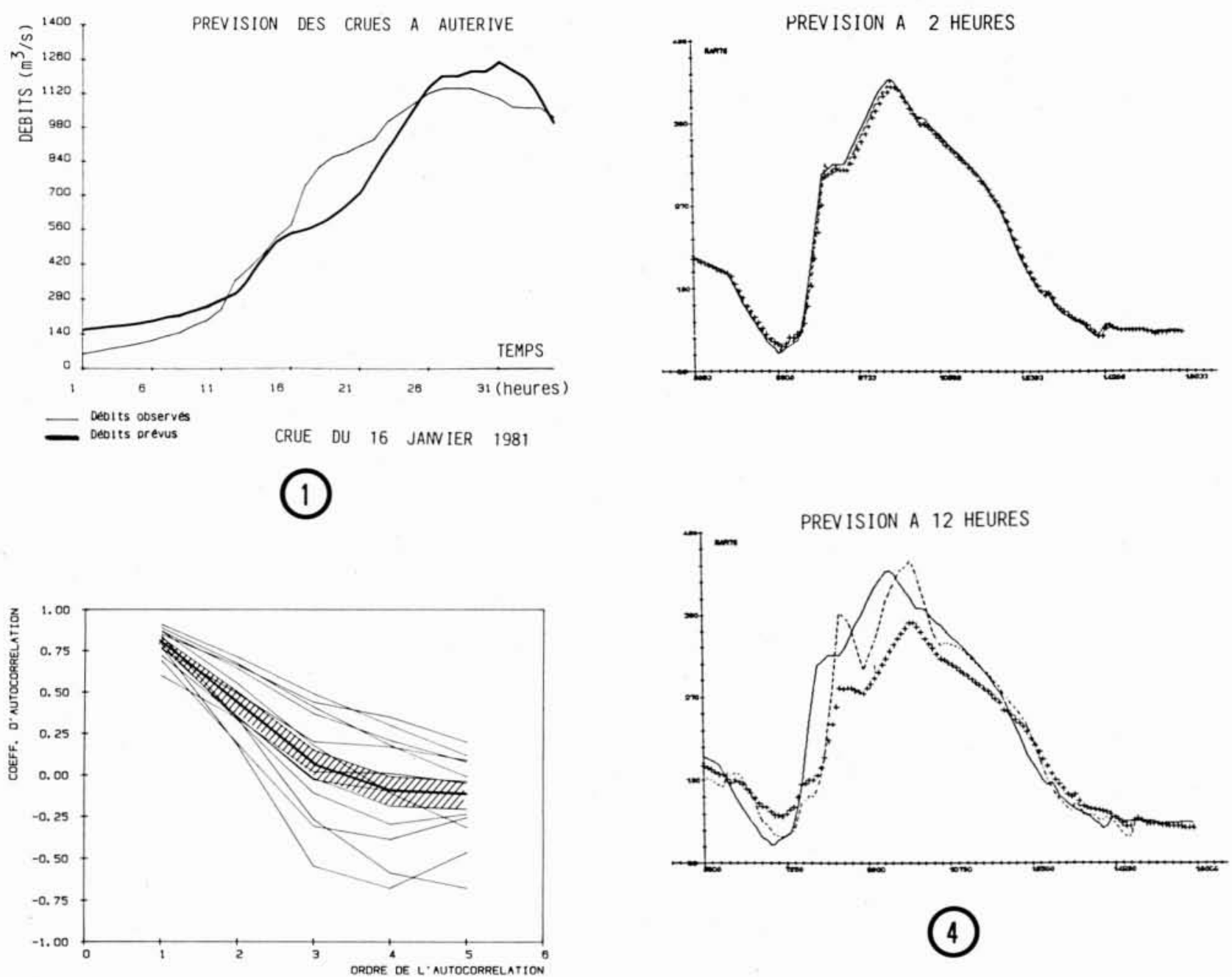

(2)

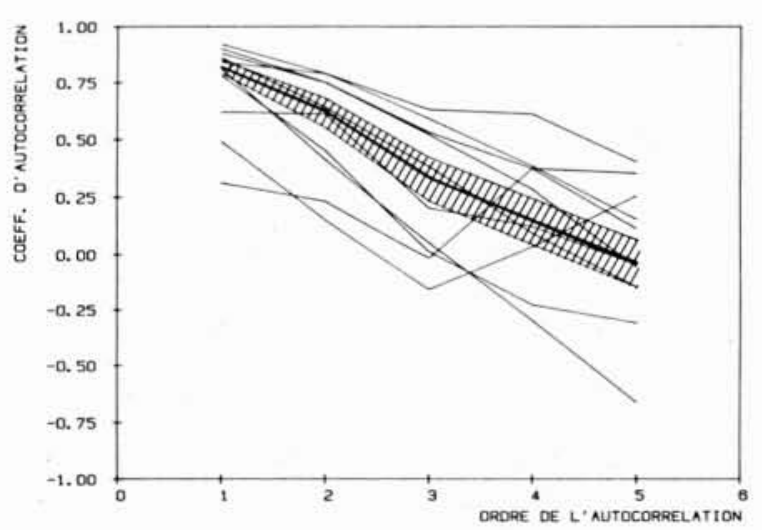

1. Hétérogénéité des écarts de prévision en cours de crue. Prévision à Auterive (bassin de la Garonne supérieure).

2. Autocorrélogramme des écarts de prévision calculés pour chaque crue. En hachuré, le domaine de confiance de l'au. tocorrélogramme calculé sur l'ensemble de la période.

Cas de Roquefort : les autocorrélations de faible rang apparaissent bien homogènes.

3. Autocorrélogramme des écarts de prévision calculés pour chaque crue. En hachuré, le domaine de confiance de l'au. tocorrélogramme calculé sur l'ensemble de la période.

Cas de Saint-Martory: on observe une forte dispersion des autocorrélations calculées crue par crue, y compris pour les faibles ordres.

4. Oscillations induites par la correction d'écarts. Prévision des crues à Morsang-sur-Orge. Effet mis en évidence par L. Lichtiger (1987).

- $-\ldots$ débits prévus avec traitement des écarts 
parmi toutes les corrections possibles.

Cette technique de correction présente les caractéristiques suivantes :

- elle ne modifie pas le fonctionnement du modèle;

- son calage n'interagit pas avec les paramètres du modèle (il existe par ailleurs des méthodes permettant de construire des relations linéaires prenant en compte une corrélation des écarts: les modèles linéaires à modèle d'erreur, Bastin, 1983).

On voit donc que l'hypothèse d'une structure temporelle des écarts bien définie, si elle n'est pas indispensable à la constitution d'une correction efficace, est essentielle pour le choix de la structure de cette correction. Nous allons discuter ici 2 points particuliers :

- peut-on supposer que le processus des écarts a une structure homogène?

- pourquoi se restreint-on dans les applications à des processus stationnaires de type $\operatorname{ARMA}(p, q)$, sans exploiter les possibilités de dérive des processus non stationnaires ARIMA $(p, 1, q)$ ?

\subsection{Effet de l'hypothèse d'homogénéité du pro- cessus des écarts}

Existe-t-il une permanence dans les dépendances temporelles des écarts successifs en toutes circonstances?

On observe couramment l'existence d'une hétérogénéité dans les écarts-types d'erreurs (Lebossé, 1983) qui conduit à spécifier la qualité des résultats selon que l'on est en montée, en descente de crue, au-dessus ou endessous d'un seuil...

Pour la structure d'autocorrélation, il n'est guère possible d'être aussi fin, bien que l'inspection visuelle suggère de telles hétérogénéités (figure 1). Les séquences de montée, notamment, qui concernent au plus 5 à 10 pas de temps, sont de durée insuffisante pour identifier l'autocorrélogramme jusqu'à une profondeur de 5 pas de temps, ce qui est un minimum.

En revanche, il a été possible, sur la Garonne amont (Roche et Torterotot, 1987) de calculer les autocorrélogrammes crue par crue. Les figures 2 et 3 montrent :

- dans un cas, l'ensemble des autocorrélogrammes calculés est compatible avec l'hypothèse d'un processus homogène;

- dans un autre cas, plusieurs crues ne présentent pas d'autocorrélation significative des écarts alors que d'autres en ont de très fortes.

Dans ce cas, la structure de processus identifiée apparaît plutôt comme le résultat moyen du mélange de types de structures variées.

Les écarts importants, qui sont liés à une insuffisance des données ou de la modélisation pour les crues mal représentées, sont également ceux qui présentent les plus grandes persistances. De ce fait, ils contribuent fortement au calcul de l'autocorrélation, comme au calcul de la dispersion. Le traitement d'écart se règle donc spontanément, non pas pour traiter les bruits résiduels des bonnes prévisions, mais pour faire face aux défauts des crues les plus mal représentées. La procédure, jugée sur la réduction des écarts-types de prévision, est efficace : elle agit de façon optimale sur les écarts les plus forts.
Mais, en surestimant ainsi la correction à effectuer sur les crues bien modélisées, on introduit sur celles-ci des oscillations dans les résultats successifs. Même si ces oscillations ne sont pas très graves en terme d'écart-type, elles perturbent le travail du prévisionniste. En effet, elles détruisent la structure d'évolution de la crue: création de maxima artificiels, dont le prévisionniste devra se méfier, difficulté à apprécier les temps de réponses, etc. (figure 4).

Le bénéfice du traitement d'écart est donc à discuter : pour de fortes autocorrélations, il masque le modèle et améliore les performances (mais s'il y a de fortes persistances des écarts, c'est que le modèle est mauvais). Pour des autocorrélations inférieures à 0.6 , la réduction de l'écart-type est inférieure à $20 \%$ : cela ne présente plus guère d'intérêt. Le créneau intéressant est somme toute assez étroit. Mais, avec des précautions d'emploi opérationnel, la procédure ne présente pas grand danger.

\subsection{Erreurs stationnaires ou non stationnai- res?}

A notre connaissance, toutes les applications de traitement d'écart en prévision des crues ont fait l'hypothèse que les écarts étaient stationnaires (processus ARMA $(p, q))$. C'est bien compréhensible dans l'hypothèse qu'il s'agit d'un petit bruit résiduel, c'est-à-dire si le modèle est entièrement correct. On considère généralement que si des dérives apparaissent, c'est le filtrage qui est l'outil le mieux adapté à la prise en compte de ces dérives.

Une structure ARMA $(p, q)$ intégrée une fois (ARIMA $(p, 1, q))$ décrit très bien un processus non stationnaire : pourquoi ne pas l'utiliser lorsque l'on suspecte la possibilité d'un détarage du modèle, ou lorsque l'on observe qu'en cours de crue les erreurs semblent se cumuler dans des décalages systématiques, mais particuliers à chaque épisode (cas classique des apports intermédiaires mal contrôlés) ?

Sur l'un des exmeples cités dans le Guide de prévision des crues (p. 51), nous avons appliqué ce principe (Roche, Tamin, 1986) : en effet la relation entre hauteurs calculées et hauteurs observées traduisait, par le dessin de raquettes, un tel comportement des écarts.

Pour la relation:

$$
y_{i+1}=f\left(x_{i}\right)+e_{i+1}
$$

$y$ : cote à la station de Montignac

$x$ : cote à la station de Larche

$i$ : indice du pas de temps (ici 4 heures)

la dispersion des écarts était de $20 \mathrm{~cm}$.

Nous avons étudié les autocorrélations des variations d'écart:

$$
e_{i+1}^{\prime}=e_{i+1}-e_{i} \text { (figure 5, page suiv.). }
$$

Pour des raisons explicitées au paragraphe 2, nous avons ajusté une structure ARMA $(1,1)$ à ce processus :

$$
e_{i+1}^{\prime}-b e_{i}^{\prime}=u_{i+1}-c u_{i}
$$

avec $u_{i}$ bruit blanc.

Nous avons obtenu $b=0.71$ et $c=0.18$, un écart-type pour $u$ de $5,6 \mathrm{~cm}$ et nous avons vérifié que $u$ était bien un bruit blanc. 


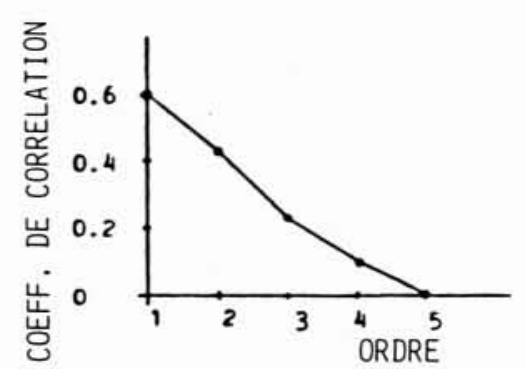

\section{5 . Autocorrélogramme des variations d'écarts.} Prévision sur Montignac (Vézère). $Y_{i+1}$ :

Une telle structure de processus conduit à la prévision

$$
\begin{aligned}
Y_{1}= & f\left(x_{0}\right) \\
Y_{i+1} & =f\left(x_{i}\right)-c\left[y_{i}-Y_{i}\right] \\
& +(1+b)\left[y_{i}-f\left(x_{i-1}\right)\right]-b\left[y_{i-1}-f\left(x_{i-2}\right)\right]
\end{aligned}
$$

Cette correction, de structure en apparence plus complexe que celles utilisées habituellement, ne présente aucune difficulté de calcul, puisque les valeurs $b$ et $c$ se déduisent immédiatement des autocorrélations de $e_{i}^{\prime}$.

\subsection{Processus d'écarts non stationnaire et méthode des variations des variables}

Une autre pratique pour écarter la question de la non stationnarité sans pour autant filtrer les paramètres du modèle est d'ajuster des modèles dont les variables sont les accroissements de débits ou de cotes

$$
y_{i+1}-y_{i}=g\left(x_{i}\right)+\varepsilon_{i+1}
$$

(les $x_{i}$ pouvant eux-mêmes être des variations).

Le modèle Arima précédent permet la formulation suivante :

$$
y_{i+1}-y_{i}=f\left(x_{i}\right)-f\left(x_{i-1}\right)+u_{i}-c u_{i-1}
$$

qui fait directement un modèle aux différences, mais dans un cas où le bruit a subi une moyenne mobile.

En dehors de la différence sur la structure des bruits, on pourra remarquer que l'emploi du modèle $(g)$ aux différences pose du fait de son mode d'identification le problème d'une dérive non contrôlée si on l'emploie de façon itérative en simulation, alors que le modèle $(f)$ fournit une prévision sans dérive si celle-ci n'est pas constatée par des observations intermédiaires.

\section{Filtrage}

\subsection{Rappel sur les objectifs du filtrage}

Si les études de méthodes de prévision des crues utilisant un filtrage des paramètres des modèles abondent, on

serait bien en peine d'en trouver qui aient été mises en œuvre dans un service opérationnel. Cela provient sans aucun doute de la réticence des utilisateurs à voir une procédure automatique modifier les paramètres de leurs modèles, et de la crainte qu'ils ont de rapidement ne plus savoir où ils en sont. Ainsi qu'il est expliqué dans le guide de prévision des crues, ces craintes sont peu fondées, et cette méthode est sans aucun doute un moyen efficace de maintenance des modèles. Nous allons ajouter quelques réflexions sur la façon dont le filtrage se place vis-à-vis du traitement des écarts.

On peut être surpris lorsqu'on lit les études récentes utilisant le filtrage. Celui-ci est clairement destiné à prendre en compte les possibilités de détarage des modèles. Pour le caler, il faut donc disposer d'événements sur lesquels un détarage du modèle a effectivement été constaté : cela a été le cas pour la première étude de J. Miquel sur la Loire à Tours (Miquel, 1982). Mais depuis, nombreux sont ceux qui ont calé des filtres sur des cas ne présentant pas de non-stationnarité manifeste.

Alors le filtrage devient un moyen de faire face aux écarts présentant une persistance de plusieurs pas de temps. En faisant l'hypothèse "classique " de résidus blancs pour le modèle filtré, on contraint - au calage - le filtre à réagir aux écarts de prévision à très court terme. Par contre, si on le destine à répondre aux dérives des paramètres à moyen terme, on tolère une certaine persistance d'écarts qu'il faut alors prendre en compte par le traitement d'écarts (qui par nature agit à très court terme). On évite ainsi d'avoir un filtre qui fait évoluer les paramètres des modèles à chaque perturbation pour leur redonner leurs valeurs initiales peu après la perturbation (voir Guide de prévision des crues, annexe VIII.1).

Nous avons donc été conduits à étudier 3 aspects particuliers du filtrage :

- le filtrage avec un bruit d'observation autocorrélé;

- le lien entre filtrage et traitement d'écarts;

- le choix de l'échantillon de calage.

\subsection{Filtrage avec un bruit d'observation autocorrélé}

Le filtrage de Kalman s'intéresse au système :

équation d'évolution: $x_{i+1}=f_{i} \cdot x_{i}+e_{i}$

équation d'observation : $y_{i}=c_{i} \cdot x_{i}+u_{i}$

Nous avons développé les raisons qui nous poussent à considérer un bruit d'observation $u_{i}$ autocorrélé. On suppose alors qu'il s'agit d'un processus autorégressif de rang 1 :

$$
u_{i}=r \cdot u_{i-1}+v_{i} \quad\left(v_{i} \text { bruit blanc }\right)
$$

Une première réponse indiquée dans le guide de prévision des crues, citant de façon un peu rapide Radix (1970) est d'augmenter la taille du vecteur d'état en y ajoutant le bruit $u_{i}$. Mais alors le système n'est plus observable au sens de Kalman et ne peut être résolu.

Bryson et Henrikson (1968) proposent une approche qui consiste à modifier la variable d'observation $y_{i}$ en $y_{i}-r \cdot y_{i-1}$, et ils donnent l'expression des équations 


\begin{tabular}{|l|l|l|l|}
\hline RHO & EQM & REA & RES \\
\hline 0.00 & 41.4 & 1.00 & 0.01 \\
\hline 0.10 & 41.4 & 1.00 & 0.01 \\
\hline 0.20 & 41.4 & 0.99 & 0.05 \\
\hline 0.30 & 41.4 & 1.00 & 0.03 \\
\hline 0.40 & 41.3 & 1.01 & 0.03 \\
\hline 0.50 & 41.1 & 1.01 & 0.08 \\
\hline 0.60 & 40.7 & 0.99 & 0.14 \\
\hline 0.70 & 40.5 & 1.00 & 0.08 \\
\hline 0.80 & 40.6 & 0.99 & 0.06 \\
\hline 0.90 & 40.7 & 1.00 & 0.01 \\
\hline
\end{tabular}

Tableau 1a

\begin{tabular}{|l|l|l|l|}
\hline RHO & EQM & REA & RES \\
\hline 0.00 & 68.5 & 1.00 & 0.72 \\
\hline 0.10 & 67.7 & 0.99 & 0.76 \\
\hline 0.20 & 67.5 & 1.00 & 0.75 \\
\hline 0.30 & 66.6 & 1.00 & 0.75 \\
\hline 0.40 & 66.3 & 1.00 & 0.73 \\
\hline 0.50 & 64.8 & 1.00 & 0.73 \\
\hline 0.60 & 64.3 & 1.02 & 0.70 \\
\hline 0.70 & 63.8 & 1.01 & 0.68 \\
\hline 0.80 & 65.0 & 1.00 & 0.65 \\
\hline 0.90 & 66.6 & 1.00 & 0.63 \\
\hline
\end{tabular}

Tableau 1b

\begin{tabular}{|l|l|l|l|}
\hline RHO & EQM & REA & RES \\
\hline 0.00 & 42.8 & 1.01 & 0.84 \\
\hline 0.10 & 42.3 & 1.03 & 0.82 \\
\hline 0.20 & 41.6 & 1.00 & 0.82 \\
\hline 0.30 & 40.9 & 0.99 & 0.82 \\
\hline 0.40 & 40.2 & 0.97 & 0.82 \\
\hline 0.50 & 39.4 & 0.94 & 0.81 \\
\hline 0.60 & 38.9 & 1.00 & 0.81 \\
\hline 0.70 & 37.9 & 1.00 & 0.80 \\
\hline 0.80 & 37.6 & 0.98 & 0.81 \\
\hline 0.90 & 36.6 & 0.97 & 0.83 \\
\hline 0.95 & 37.1 & 0.91 & 0.80 \\
\hline & & & \\
\hline
\end{tabular}

Tableau 1c

gouvernant le filtre. Mais cette approche suppose le coefficient $r$ fixé, alors qu'il n'est pas déterminable ici a priori (pour chaque valeur de $r$ on peut caler un filtre par le choix des matrices de covariance de $e_{i}$ et $v_{i}$ ).

Cette approche a été mise en œuvre sur des chroniques de débits (1000 enregistrements) pour lesquelles on a simulé une dérive aléatoire des paramètres d'un modèle de prévision, avec des bruits d'observation $u_{i}$ autocorrélés (ici $r_{\text {réel }}=0.7$ ). Pour différents coefficients d'autocorrélation $r$ pris en compte, on a calé le filtre sur deux critère indépendants (Roche et Torterotot, 1987) :

- l'erreur de prévision quadratique moyenne;

- le critère de réalisme qui mesure l'adéquation entre performances réelle et théorique du modèle filtré (la formule de prévision intègre le traitement autorégressif).

On a pu mettre au point une procédure automatique de calage du filtre.

A deux échéances de prévisions différentes (1 et 3 pas de temps), on a obtenu la meilleure prévision en prenant en compte la véritable valeur du coefficient d'autocorrélation $r_{\text {réel }}=0.7$ (tableaux $1 a$ et $\left.1 b\right)$. Cette vérification pratique de la théorie n'apparaît toutefois pas convaincante en ce qui concerne l'amélioration significative de la prévision opérationnelle.

C'est pourquoi la procédure a été mise en œuvre sur une série de crues réelles (cas de la Garonne supérieure, 700 enregistrements) comportant un détarage caractérisé. Le tableau $1 c$ présente les résultats qui se révèlent être plus intéressants que sur les chroniques simulées. En prenant en compte un coefficient $r=0$ (filtre " classique "), on obtient un écart de prévision quadratique moyen de $42.8 \mathrm{~m}^{3} / \mathrm{s}$ au lieu de $36.6 \mathrm{~m}^{3} / \mathrm{s}$ pour $r=0.9$ (perte de précision de $17 \%$ due à la sous-optimalité du filtre classique).

L'intérêt de la procédure du filtrage prenant en compte l'autocorrélation des bruits d'observation devra être confirmé par des mises en œuvre ultérieures avant que cette approche ne soit proposée de façon systématique.
Tableau 1a : Performances d'un modèle linéaire filtré avec prise en compte de différentes valeurs pour le coefficient d'autocorrélation $\mathrm{RHO}$ des bruits d'observation.

Mise en œuvre sur une série simulée de 1000 enregistrements avec $\mathrm{RHO}_{\text {réel }}=0.7$, prévision à échéance de 1 pas de temps.

RHO : coefficient d'autocorrélation pris en compte par le filtre

EQM : écart quadratique moyen de prévision en $\mathrm{m}^{3} / \mathrm{s}$

REA : critère de réalisme

RES : coefficient d'autocorrélation des résidus du modèle filtré

Tableau 1b : Performances d'un modèle linéaire filtré avec prise en compte de différentes valeurs pour le coefficient d'autocorrélation $\mathrm{RHO}$ des bruits d'observation.

Mise en œuvre sur une série simulée de 1000 enregistrements avec $\mathrm{RHO}_{\text {réel }}=0.7$, prévision à échéance de 3 pas de temps.

RHO : coefficient d'autocorrélation pris en compte par le filtre

EQM : écart quadratique moyen de prévision en $\mathrm{m}^{3} / \mathrm{s}$

REA : critère de réalisme

RES : coefficient d'autocorrélation des résidus du modèle filtré

Tableau 1c: Performances d'un modèle linéaire filtré avec prise en compte de différentes valeurs pour le coefficient d'autocorrélation $\mathrm{RHO}$ des bruits d'observation.

Mise en œuvre sur une série réelle de 700 enregistrements, prévision à échéance de 3 pas de temps des débits à la station de Saint-Martory en fonction des débits à Valentine (Garonne supérieure).

RHO : coefficient d'autocorrélation pris en compte par le filtre

EQM : écart quadratique moyen de prévision $\mathrm{en} \mathrm{m}^{3} / \mathrm{s}$

REA : critère de réalisme

RES : coefficient d'autocorrélation des résidus du modèle filtré 


\subsection{Lien entre filtrage et traitement des écarts}

Le développement précédent, où les identifications du filtre et de la structure des écarts sont liées, et l'introduction des structures d'écart non stationnaires (1.3) montrent que les relations entre filtrage et traitement d'écart ne sont pas simple.

En fait, nous avons pu montrer l'équivalence du filtrage d'un simple décalage et d'un traitement d'écart non stationnaire (Roche et Tamin, 1986, dans le cas sans autocorrélation, Roche et Torterotot, 1987 en cas d'autocorrélation).

Reprenons la formulation de la relation (4) en considérant que le modèle $f$ comporte une composante additive $a_{i}$ et que l'on cherche à filtrer cette constante.

$$
\begin{array}{cc}
\left.\qquad x_{i}\right)=f_{0}\left(x_{i}\right)+a_{i} & \\
a_{i+1}=a_{i}+t_{i} & \text { évolution } \\
y_{i+1}=f_{0}\left(x_{i}\right)+a_{i}+v_{i} & \text { observation }
\end{array}
$$

où $t_{i}$ et $v_{i}$ sont des bruits stationnaires.

L'autocorrélation des écarts à court terme (indépendamment de l'évolution de la composante $a_{i}$ ), se traduit par une relation :

$$
v_{i}=r v_{i-1}+w_{i}
$$

où $w_{i}$ est un bruit blanc gaussien.

Le système (10) (11) étant stationnaire, le filtrage se fait avec un gain $\mathrm{k}$ constant.

Il existe alors les relations suivantes :

- si le filtrage est optimal, ses innovations $\left(u_{i}\right)$ (écarts de prévision après filtrage) sont un bruit blanc, qui est relié aux écarts du modèle non filtré $\left(e_{i}\right)$ par la relation :

$$
\begin{gathered}
e_{i}^{\prime}=e_{i}-e_{i-1} \\
e_{i}^{\prime}-r e_{i-1}^{i}=u_{i}-(1-(1-r) k) u_{i-1}
\end{gathered}
$$

Le processus des écarts du modèle non filtré est alors un ARIMA $(1,1,1)$ (cf. (5)) donc les caractéristiques sont :

$$
\begin{aligned}
& (b=r) \\
& (c=1-(1-r) k)
\end{aligned}
$$

Pour $r=0$, le processus est un $\operatorname{IMA}(1,1)$;

- si l'on a identifié un tel processus, on en déduit le gain du filtre $k$ et l'autocorrélation $r$ des écarts d'observation $\left(v_{i}\right)$ en utilisant (14), ce qui est beaucoup plus pratique que l'analyse de sensibilité des variances des bruits d'évolution et d'observation du filtre. Par contre, si le processus n'est pas identifiable, il reste possible de caler un filtre.

Nous proposons donc, dès lors que l'on envisage de filtrer les paramètres d'un modèle, d'étudier préalablement le traitement d'écart instationnaire, qui, correspondant à une version simplifiée du filtrage, permet une première analyse de la dérive envisagée.

Un filtrage sans prise en compte de l'autocorrélation, après optimisation des variances des bruits, a conduit au résultat suivant pour le cas traité au chapitre précédent :

- écart-type des innovations : $7.3 \mathrm{~cm}(+30 \%)$;
- autocorrélation des innovations : 0.5 (théoriquement 0 .);

- coefficient de gain : 0.9993 (saturation à 1).

\subsection{Choix des données de calage}

Que ce soit pour un traitement d'écart non stationnaire ou pour un filtrage plus complet, rien n'assure qu'il existe dans l'échantillon des données historiques les événements susceptibles d'en activer le fonctionnement. Faut-il pour autant renoncer à prendre en compte pour l'avenir l'éventualité d'événements conduisant à une dérive ? Certainement pas. La mise en œuvre opérationnelle est tournée vers le futur, non vers le passé.

On peut donc être conduit à simuler des événéments fictifs superposés aux événements observés, afin de tester, ou de caler ces dispositifs (Roche et Torterotot, 1987).

\section{Procédures multimodèles}

\subsection{Objectifs}

Les systèmes présentés ci-dessus n'utilisent que les écarts d'un modèle: en temps réel, une information beaucoup plus riche peut être utilisée en exploitant différentes combinaisons des indicateurs télétransmis, en comparant les résultats des modèles qui les utilisent et en analysant la cohérence de ces diverses informations.

On peut ainsi faire face:

- à des perturbations inhabituelles sur certains indicateurs (dégradation de l'information);

- à des situations nouvelles, pour lesquelles l'absence d'information historique n'a pas permis de déterminer la structure du modèle (fonctionnement des modèles en extrapolation).

Le plus simple est de choisir parmi les modèles celui qui fournit le meilleur résultat depuis quelques temps: une démarche hiérarchique proposée par Lebossé et Miquel (1985) permet de stabiliser ces choix.

Dans d'autres domaines que la prévision, Vernos (1982) a développé une analyse bayésienne de ces décisions de changement de modèles, et Cavadias et Morin (1986) ont proposé une combinaison linéaire des modèles.

L'approche qui sera présentée ici est une combinaison de modèles par pondération, les poids de chaque modèle évoluant en fonction des configurations du système modélisé et des performances récentes des modèles. Deux études sont fondées sur cette approche, l'une sur la Vézère (Roche et Tamin, 1986), l'autre sur la Garonne supérieure (Roche et Torterotot, 1987). La figure 6 présente pour ces deux cas les cartes des bassins versants avec les stations.

\subsection{Principe}

Le problème de temps réel se décompose en 2 étapes qu'il ne faut pas confondre: 
6. Cartes des bassins de la Garonne supérieure (amont de Toulouse) et de la Vézère.

7. Configurations pour deux situations typiques.

Premier cas : données correctes (C) ou erronées (E) sur les principales stations de prévision; exemple tiré de la prévision à Montignac (bassin de la Vézère)

Deuxième cas : aux perturbations précédentes s'ajoute l'incertitude quant au comportement des crues fortes sur deux affluents amont : crues fortement débordantes $(D)$, ou dont les débordements ne conduisent pas à une grande modification des caractéristiques de propagation (ND) exemple tiré de la Garonne supérieure

- une étape de diagnostic: il s'agit avec toutes les informations collectées, de faire le point de la cohérence de celles-ci et d'essayer d'en tirer des conclusions portant ou bien sur les modèles, ou bien sur les données. Bien entendu, cette étape, que l'on suppose lors de l'étude réalisée de façon automatique à partir de divers tests peut être complétée en temps réel par les éléments d'appréciation du prévisionniste et des informations extérieures au système de collecte automatique;

- une étape de pronostic: le diagnostic étant fait, il s'agit de l'utiliser au mieux pour prévoir les débits ou les niveaux à l'aval. Pour cela, il y a généralement intérêt non pas à choisir une hypothèse ou un modèle particulier, mais à utiliser une pondération entre les différents résultats.

On suppose que les écarts de prévision de chaque modèle ne présentent pas d'autocorrélation, sans quoi on peut effectuer préalablement les traitements évoqués ci-dessus (à noter d'ailleurs qu'une version de cette procédure multi-modèle intégrant directement les autocorrélations est tout-à-fait possible; il a semblé ici plus simple de séparer cohérence temporelle et cohérence entre modèles au même pas de temps).

On dispose de $m$ modèles $M_{k}(k$ de 1 à $m$ ) fournissant respectivement les prévisions $y_{k}$ de la grandeur $Y$ à prévoir. Cela nous conduit à proposer une prévision :

$$
y=\sum_{i=1}^{m} w_{k} y_{k} \quad\left(\Sigma w_{k}=1\right)
$$

Le poids $w_{k}$ est constitué d'une composante stationnaire et d'une composante évolutive :

$w_{k}=\beta \cdot w_{k}$ (stationn.) $+(1-\beta) \cdot w_{k_{t}}^{1}$ (évolut.)

\subsection{Pondération stationnaire}

On suppose que le système modélisé (hydrologie et réseau de transmission) peut se trouver dans $n$ différentes configurations - ou hypothèses de fonctionnement $-H(i)$ ( $i$ de 1 à $n$ ). La figure 7 présente des configurations identifiées sur les deux études de prévision citées plus haut. Nous reviendrons plus loin sur l'identification de configurations dites "donnée erronée " et "expansion ".
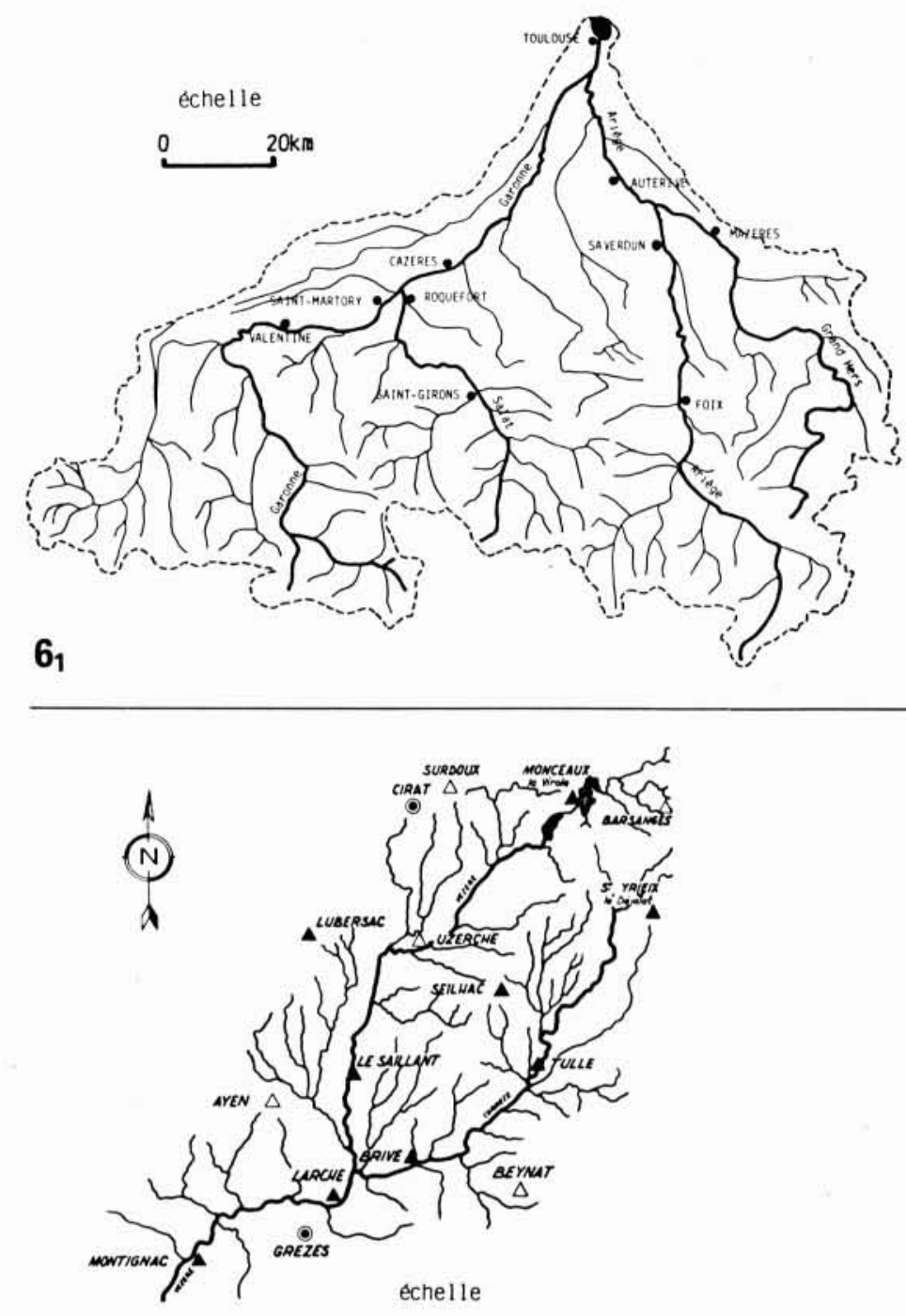

$6_{2}$

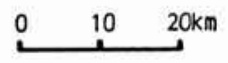

VEZERE

GARONNE SUPERIEURE

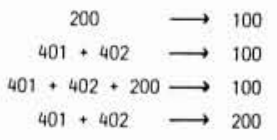

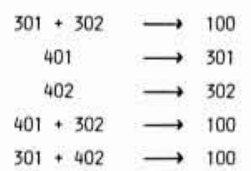

7.
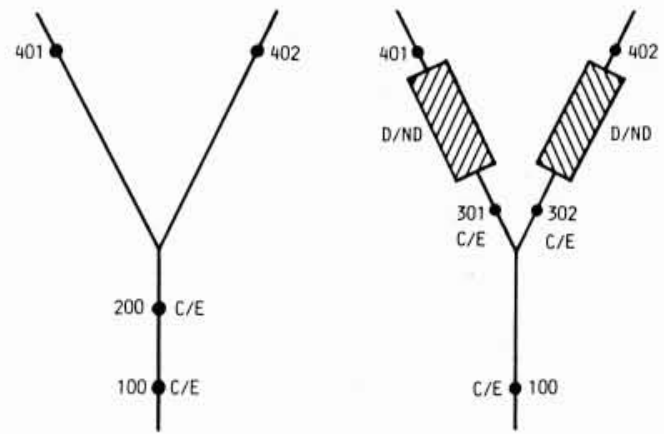


\subsubsection{Prévision pour une configuration déterminée}

On s'intéresse donc d'abord au cas où il n'y a qu'une configuration. C'est par exemple celui de la mise au point en mode normal d'une méthode de prévision à partir de différents résultats de modèles.

La méthode utilisée a été proposée pour la prévision économique par Newbold et Granger (1974). Il s'agit essentiellement d'une combinaison des valeurs prévues utilisant la covariance des écarts de prévision $V$ supposée connue.

Soit :

- $y$, la variable à prévoir (la configuration $H$ étant donnée). C'est l'état du système qu'on cherche à estimer; $-\left\{y_{k}\right\}$, les prévisions fournies pour les différents modèles $M_{k}, k=1 \ldots m$. Ce sont les informations dont on dispose.

On veut estimer la variable $y$ sur la base des informations $\left\{y_{k}\right\}$. On suppose que les écarts $e_{k}=y_{k}-y$ (pour tout $k$ ) suivent une loi normale de dimension $m$ :

$$
\left\{e_{k}\right\}: \mathscr{N}^{(m)}(0, V),
$$

$V=$ matrice de covariance des écarts des prévisions.

On cherche une estimation de $y$. C'est une décision $d\left\{y_{k}\right\}$, qui minimise un critère $C\left(d\left\{y_{k}\right\}\right)$ de proximité à $y$ qu'on peut raisonnablement prendre quadratique :

$$
C\left(d\left\{y_{k}\right\}\right)=E\left[\left(y-d\left\{y_{k}\right\}\right)^{2}\right]
$$

La décision optimale est alors l'espérance conditionnelle $:$ dopt $=E\left(y\left\{y_{k}\right\}\right)$

L'application simple de la règle de Bayes (Winkler, 1981) en supposant que la loi de distribution a priori de $y$ est diffuse (impropre) permet de montrer que la densité de probabilité de $y$ connaissant $\left\{y_{k}\right\}$ suit une loi normale de moyenne $M=n^{t} \cdot V^{-1} \cdot Y / n^{t} \cdot V^{-1} \cdot n$ et de variance $U=1 / n^{t} \cdot V^{-1} \cdot n$, avec $n=(1 \ldots .1)^{t}$ et $Y=\left(y_{1} \ldots y_{k} \ldots y_{m}\right)$.

D'où l'expression :

$$
\begin{gathered}
\operatorname{dopt}\left\{y_{k}\right\}=\sum_{k} w_{k} \cdot y_{k} \\
w_{k}=\sum_{\ell} \alpha(k, \ell) / \sum_{k \ell} \alpha(k, \ell)
\end{gathered}
$$

où $\{\alpha(k, \ell)\}=V^{-1}$

On appelle $w_{k}$ le poids accordé au modèle $k$.

La somme des $w_{k}$ est égale à 1 , mais le poids d'un modèle peut être négatif si les écarts des modèles concernés sont fortement corrélés (positivement) entre eux.

On remarquera que la variance $U$ est inférieure à la variance du meilleur modèle dans tous les cas. Ainsi la décision optimale ne consiste pas à choisir le meilleur modèle, contrairement à ce qui est supposé implicitement dans les études de prévision. Néanmoins, tant qu'il s'agit de conserver une telle pondération stationnaire, l'intérêt de cette démarche reste limité. On obtient en effet par pondération un nouveau modèle, comportant beaucoup plus de paramètres que chacun des modèles initiaux, et dont l'interprétation est plus délicate. On trouvera dans (Roche, Tamin, 1986) une discussion détaillée de ce point.

\subsubsection{Procédure de décision dans le cas où les probabilités des configurations $H(i)$ sont connues}

De la même manière que précédemment, considérons :

- l'état du système : $y$

- les informations: $\left\{y_{k}\right\}$

On introduit maintenant: $p(H(i))=$ probabilité d'être dans la configuration $H(i)$. Pour chacune des configurations $H(i)$, on suppose identifiée la structure des écarts des prévisions :

$$
\left\{e_{k}\right\}_{H(i)} \in \mathscr{N}^{(m)}(0, V(i))
$$

L'estimation de $y$ est calculée à partir de la probabilité composée :

$$
p\left(y \mid p(H(i)),\left\{y_{k}\right\}\right)=\sum_{i} p(H(i)) \cdot p\left(y \mid H(i),\left\{y_{k}\right\}\right)
$$

La décision optimale devient maintenant :

dopt $=E\left(y \mid\left\{y_{k}\right\}, p(H(i))\right)$

dopt $=\sum_{k} w_{k} \cdot y_{k}$

d'où : $\quad w_{k}=\sum_{i} p(H(i)) \cdot w_{k}(i)$

où $w_{k}(i)$ est le poids qu'aurait le modèle $k$ dans la configuration $H(i)$.

On remarquera que la décision optimale est une combinaison linéaire des décisions prises dans chaque configuration, pondérées par la probabilité de chaque configuration.

Ce résultat élémentaire contredit également la pratique habituelle qui consiste à d'abord faire un choix de configuration, puis à utiliser le meilleur modèle dans cette configuration (approche multi-modèle, Vernos, 1982).

Cette approche par pondération stationnaire permet de s'adapter aux types de fonctionnement du système identifiés en valorisant conjointement la cohérence existant entre les modèles (dans une configuration donnée) et des informations extérieures aux modèles, permettant de préciser les probabilités d'être dans chaque configuration.

Revenons sur l'identification des configurations et de leurs probabilités.

Pour les configurations "donnée erronée ", on s'intéresse à des états discrets. Ceci permet d'envisager un diagnostic relativement facile. Le diagnostic se fait à partir des données disponibles à la date de prévision, à partir de tests de cohérence exploitant les relations établies entre stations amont et aval.

Les tests sont composés afin d'éliminer les ambiguïtés. Une relation entre 2 stations amont et aval permet, en examinant les erreurs de prévision antérieures, de porter un diagnostic de cohérence entre les 2 données. Deux autres tests, effectués à partir de stations plus en amont, permettent alors de compléter ce diagnostic; si une incohérence apparaît sur toutes les relations concernant un indicateur et non sur les autres, celui-ci a une probabilité significative d'être en état perturbé.

Dans l'étude sur la Vézère, les perturbations ont été étudiées sur les données historiques puis modélisées. Par simulation des perturbations et en faisant varier les 
seuils (s) d'acceptation et de rejet des tests, on obtient :

- le risque de première espèce $\alpha(\mathrm{s})$ : la probabilité d'avoir conclu du test que la donnée est erronnée alors qu'elle est bonne;

- le risque de deuxième espèce $\beta(s)$ : la probabilité d'avoir conclu du test que la donnée est bonne alors qu'elle est en fait erronée.

Ce sont ces risques qui permettent d'estimer les probabilités pour chaque configuration.

Concernant les configurations "expansion", il ne nous est pas possible de définir des configurations correspondant à des fonctionnements hydrauliques ou hydrologiques observés: ce sont des comportements susceptibles d'intervenir au-delà du domaine des observations disponibles, et notre connaissance des phénomènes physiques se limite à l'observation de leurs effets sur les données collectées.

En simulant des crues fortes avec augmentation des délais de propagation, on peut identifier des seuils à partir desquels les performances de différents modèles évoluent sensiblement. En intégrant ces configurations aux procédures multi-modèles, on rend ces dernières aptes à réagir aux situations simulées ou aux situations réelles qui s'en rapprochent. De la sorte, on identifie des configurations " fonctionnelles " par rapport au comportement des modèles.

\subsection{Pondération évolutive}

Dans la méthode décrite précédemment, l'inventaire des hypothèses $H(i)$ et la mise au point des tests permettant l'estimation de leurs probabilités nécessitent un travail significatif. Il en est de même pour les matrices de covariance des écarts des prévisions $V(i)$, qu'il faut identifier par simulation des perturbations. Nous avons donc cherché une méthode simplifiée pour faire évoluer les poids des modèles en respectant la démarche précédente.

Confrontés au problème de pondérer des méthodes de prévision en ignorant la matrice de covariance de leurs écarts, Newbold et Granger (1974) ont proposé et testé plusieurs procédures empiriques pour estimer de façon récursive les poids accordés aux différentes méthodes. Ces procédures sont basées sur une estimation de la covariance des écarts des modèles durant les derniers pas de temps, de façon à réduire au fur et à mesure les poids des modèles dont la qualité se dégrade au profit des modèles qui sont devenus plus performants. la relation appliquée est la suivante:

$$
w_{k, t}=\frac{\left[\sum_{s-t-v}^{t-1} e_{s}^{(k) 2}\right]^{-1}}{\left.\sum_{j=1}^{m}\left[\sum_{s=t-v}^{t-1} e^{(j)}\right) 2_{s}\right]^{-1}}
$$

$v=$ durée prise en compte pour analyser les écarts de prévision récents.

Elle se déduit de la formulation générale (17) en supposant que la matrice de covariance est diagonale (écarts indépendants entre eux). Une telle simplification est plus que nécessaire pour assurer la stabilité d'une estimation effectuée sur quelques pas de temps. Une valeur faible de $v$ signifie que l'on limite l'estimation du poids aux observations les plus récentes. A travers ce paramètre se trouve réintroduite la cohérence temporelle que nous avions écartée ci-dessus. Mais avec une grande différence: on s'intéresse ici à une permanence de la qualité de prévision, et non pas à une relation entre écarts successifs.

Cette procédure présente les avantages de ne pas nécessiter de calage, et de réagir à tous les types de perturbations en exploitant les performances récentes des modèles (ici la cohérence habituelle entre modèles est a priori détruite).

\subsection{Pondération globale}

Revenons sur l'expression générale du poids (16). La figure 8 compare les approches stationnaire et globale simplifiée (avec composante évolutive). Dans l'étude sur la Vézère, on ne considère de façon opérationnelle qu'une configuration "normale ". L'expression $(1-\beta)$ mesure alors la probabilité de ne pas être dans cet état " normal ". Pour la Garonne, plusieurs configurations sont prises en compte, et alors $(1-\beta)$ représente la probabilité d'être dans une configuration non considérée ou dans un état légèrement différent d'une configuration.

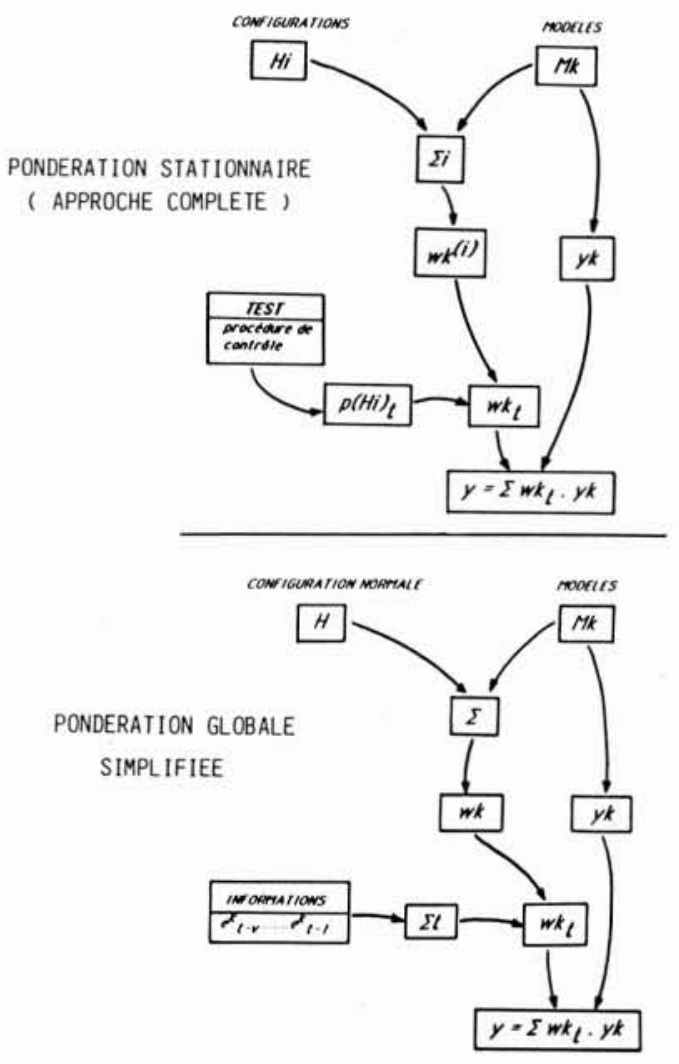

8. Principes de calcul pour la pondération stationnaire complète et pour la pondération globale simplifiée (avec pondération évolutive). 

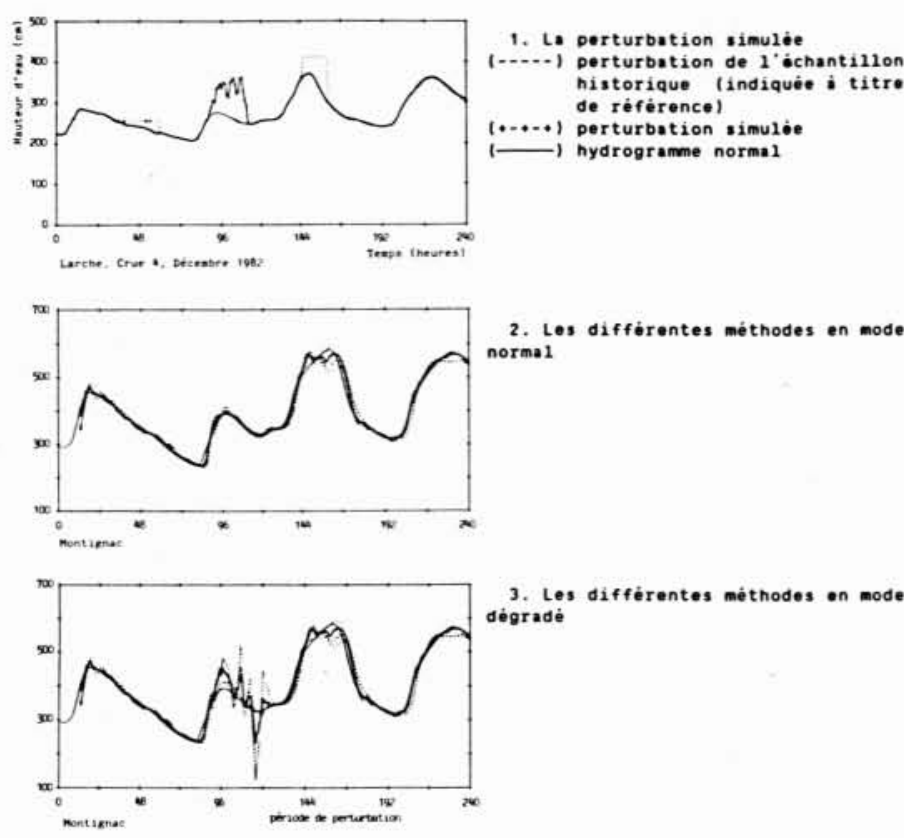

3. Les différentes méthodes on mode dégradé

2. Les differentes méthodes en mode

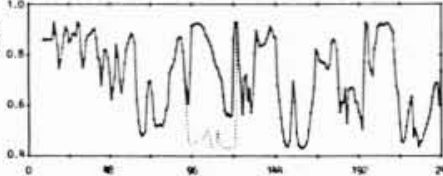

6. Evolution du poids accorde sux méthodes 1 et 2 on mode normal $(\ldots,-)$ et on mode degrade $(-. .-)$. Les poids diminuent significativement lorsque 1. station de Lerche subit une perturbation

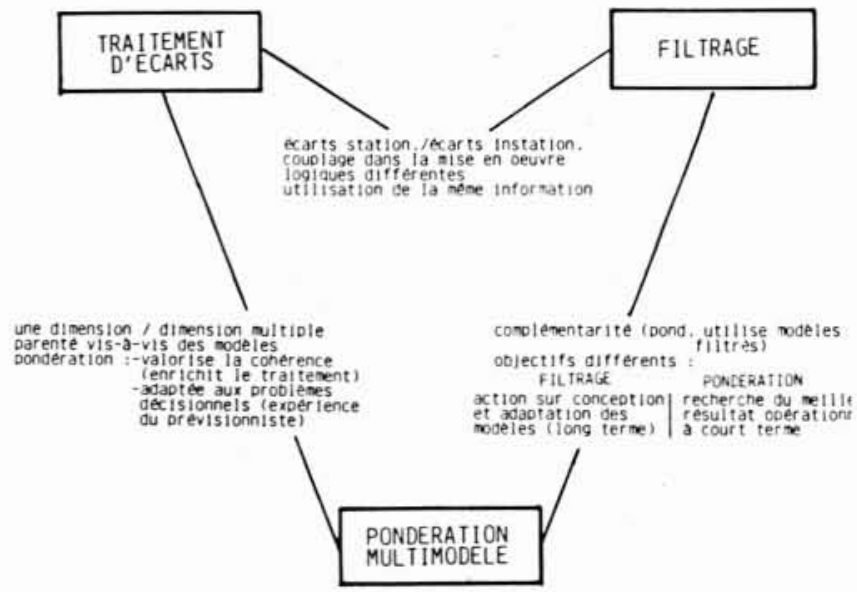

10.

9. Résultats obtenus avec la pondération évolutive (cas de la Vézère) : les poids évoluent bien pour disqualifier les méthodes les plus sensibles à la perturbation introduite.

10. Liaisons entre les trois moyens d'amélioration des performances des modèles pour le temps réel.

9.

\begin{tabular}{|c|c|c|c|c|}
\hline \multirow{3}{*}{ RELATIONS } & \multicolumn{4}{|c|}{ ERREUR QUADRATIOUE MOYENHE (CM) } \\
\hline & \multirow{2}{*}{ MODE NORHAL } & \multicolumn{3}{|c|}{ MODE DEGRADE I PERTURBATION SUR } \\
\hline & & LARCHE & MONTIGNAC & $\begin{array}{l}\text { LARCHE OU } \\
\text { MONTIGNAC }\end{array}$ \\
\hline$H=F(L)$ & 19.8 & $66.9 \pm 1.6$ & $50.2 \pm 0.7$ & $58.9 \pm 0.9$ \\
\hline$\Delta M=F(\Delta L)$ & 21.6 & $61.2 \pm 1.2$ & $38.0 \pm 0.6$ & $48.6 \pm 0.8$ \\
\hline$\Delta M=F(\Delta B, \Delta S)$ & 30.0 & $\cdot$ & $43.2 \pm 0.7$ & $43.2 \pm 0.7$ \\
\hline$\Delta M=F(\Delta M)$ & 29.1 & $\cdot$ & $62.4 \pm 0.9$ & $62.6 \pm 0.9$ \\
\hline$\Delta M=F(\Delta L, \Delta B, \Delta S)$ & 21.2 & $67.3 \pm 1.3$ & $38.6 \pm 0.6$ & $51.0 \pm 0.9$ \\
\hline PROCEDURE COMPLETE & 18.6 & $62.2 \pm 0.8$ & $37.7 \pm 0.6$ & $60.7 \pm 0.5$ \\
\hline $\begin{array}{l}\text { COMBINAISON PONDE- } \\
\text { REE EVOLUTIVE } \\
(V=3, B=0.5)\end{array}$ & 17.8 & $42.4 \pm 0.9$ & $66.2 \pm 0.6$ & $65.2 \pm 0.6$ \\
\hline $\begin{array}{l}\text { COMEINAISON PONDE - } \\
\text { REE STATIONNAIRE }\end{array}$ & 18.1 & $59.0 \pm 1.1$ & $66.5 \pm 0.6$ & $52.6 \pm 0.8$ \\
\hline COMBINAISON MOYENNE & 20.6 & $37.8 \pm 0.7$ & $64.1 \pm 0.7$ & $63.4 \pm 0.6$ \\
\hline $\begin{array}{l}\text { PASSAGE D.UN } \\
\text { MODELE A L AUTRE }\end{array}$ & 21.0 & $69.2 \pm 1.0$ & $65.1 \pm 0.7$ & $67.7 \pm 0.9$ \\
\hline $\begin{array}{l}\text { COMBINAISON PONDE - } \\
\text { REE EVOLUTIVE } \\
(V=3 . B=0)\end{array}$ & 19.2 & $34.1 \pm 1.1$ & $62.0 \pm 0.6$ & $61.7 \pm 0.6$ \\
\hline
\end{tabular}

Tableau 2. - Erreur quadratique moyenne calculée sur les seules données perturbées. Prévision à Montignac (bassin de la Vézère).

$\mathrm{M}:$ cote à Montignac $(\mathrm{t})$

$\mathrm{L}$ : cote à Larche $(\mathrm{t}-4)$

B : cote à Brive (t-8)

$\mathrm{S}$ : cote à Le Saillant $(\mathrm{t}-8)$

symbole $\Delta$ : variations des cotes 


\subsection{Résultats}

La figure 9 et le tableau 2 présentent des résultats obtenus sur la Vézère en "mode normal " et en "mode dégradé ").

La démarche globale proposée pour la Garonne supérieure, et qui sera bientôt testée, cumule les avantages des deux approches:

- utilisation de modèles de structures variées et qui ne sont pas modifiés;

- valorisation importante des performances des modèles et d'informations extérieures;

- prise en compte des mécanismes identifiés sur le système modélisé ou de mécanismes perturbateurs potentiels (par l'utilisation de la simulation);

- potentiel de réaction à tout type de perturbation.

\section{Conclusion}

Traitement d'écart, filtrage, procédures multimodèles... trois techniques permettant d'améliorer la qualité des résultats fournis en temps réel.

Plutôt que concurrents, ces moyens sont complémentaires (figure 10).

Destinés aux écarts à court terme, le traitement d'écart est nécessaire à toute autre approche : un filtrage ignorant la structure des écarts peut se révéler sousoptimal et délicat à mettre en œuvre (filtre trop verveux), et les procédures multimodèles supposent pour l'instant que les écarts sont préalablement blanchis.

Destiné à suivre l'évolution à moyen et long termes des conditions physiques de l'écoulement, le filtrage ne doit pas se substituer au traitement d'écart, et modifier à tous propos les paramètres des modèles. Mais il existe par ailleurs des liens étroits entre processus d'écarts non stationnaires et filtrage.

Outils privilégiés de la décision temps réel sur un réseau, les procédures multimodèles sont des sortes de "super-traitements d'erreurs". Complémentaires du filtrage, qui adapte les modèles à de nouvelles informations, elles se contentent, avant confirmation du sens physique de ces informations, de modifier la confiance accordée aux modèles selon la façon dont ceux-ci réagissent.

La mise au point de ces procédures diverses fait apparaitre une noouvelle logique de calage : destinées à faire face à des situations qui sont pour l'instant hypothétiques, il est nécessaire de les tester sur des scénarios possibles, et non plus seulement sur l'information historique : alors seulement la prévision acquiert une véritable sécurité de fonctionnement.

\section{Remerciements}

Les études présentées ici ont été financées par le ministère chargé de l'Environnement. Les Services hydrologiques centralisateurs de la Dordogne et de la Garonne ont aimablement communiqué les données nécessaires et assuré un suivi précieux de ces travaux.

\section{Références}

BASTIN G., 1983. - Identification de modèles de prévision du débit du Gardon d'Anduze, Rapport I.M.G.

Bryson A.E. Jr et HenriKson L.J., 1968. - Estimation using sampled data containing sequentially correlated noise, Journal of Spacecraft, vol. 5, $\mathrm{n}^{\circ} 6,662-665$.

Cavadias G. et Morin, 1985. - Amélioration des performances des modèles hydrologiques par combinaison des débits simulés, Revue Internationale des Sciences de l'Eau, vol. 1, $\mathrm{n}^{\circ} 1 / 4,29-35$.

LEBosse A., 1983. - Filtre de Kalman et annonce des crues: application au bassin du Cher, Rapport EDF 83.19.

Lebosse A. et Miquel J., 1985. - Annonce des crues du Cher: mise au point des formules de prévision, Rapport EDF E 43/84.37.

Lichtiger L., 1987. - Conception d'un logiciel de prévision des crues sur le bassin de l'Orge, Rapport de D.E.A. Techniques et Gestion de l'Environnement, Université Paris-Val-deMarne, Créteil.

Miquel, 1982. - Filtre de Kalman et annonce de crue : La Loire à Tours, Rapport EDF E43/79.48.

Newbold P. et Granger C.W.J. - Experience with forecasting univariate time series and the combination of forecast, J.R. Statist. Soc., 131-146.

RADIX, 1970. - Introduction au filtrage numérique, Eyrolles, Paris.

Roche P.A. et TAmin R., 1986. - Procédures de décision multimodèles applicables à la prévision des crues en temps réel, CERGRENE, ENPC, Noisy-le-Grand, 85 p., ann.

Roche P.A. et al., 1987. - Guide de prévision des crues, Société Hydrotechnique de France, Paris, 750 p., ann.

Roche P.A. et TorTerotot J.P., 1987. - Prévision des crues sur la Garonne supérieure : conception d'une procédure multimo. dèle, CERGRENE, ENPC, Noisy-le-Grand, 67 p., ann.

VERnos D., 1982. - Application des modèles stochastiques dynamiques linéaires à la description et à la simulation des processus de débits des rivières aux échelles saisonnières et journalières - étude de leur utilisation en prévision de la gestion des ressources en eau. Thèse de docteur de $3^{\mathrm{e}}$ cycle, Université Paris VI, Paris.

WINKLER R.L., 1981. - Combining probability distribution from dependent information sources, Management Sciences, $\mathrm{n}^{\circ} 27$, 479-488. 
La première banque de données du Bâtiment sur le réseau professionnel.

Exclusivement réservé à la promotion

de nos Annonceurs.

\section{"SAGERET BATIMENT ET T.P." \\ 175.000 Entreprises du Bâtiment \\ 25.000 Fournisseurs et Négociants \\ Accessible par le 36.14 avec mot de passe}

\section{Aurnes édition}

SERVICES Annuaire national 5 volumes (5.000 pages)

- Annuaire des Architectes

- Mémento des qualifications OPQCB

(édition 1988)

\section{mailing}

- Location de fichiers

- Routage

- Impression laser personnalisée

- Telexing 


\author{
Discussion des exposés \\ de $\mathbf{M}^{\text {me }}$ C. Cosandey (p. 381), MM. C. Thirriot (p. 394), Th. Leviandier (p. 395) et P.A. Roche (p. 399) \\ Président : M. C. FABRET
}

\begin{abstract}
Le Président: Je remercie M. TORTERotot. $\mathrm{Si}$ je devais résumer rapidement ce qui a été exposé, par les différents intervenants, je dirais que M. MAsson nous a rappelé l'importance d'un diagnostic présentant des résultats simples. $\mathrm{M}^{\text {me }}$ COSANDEY nous a dit qu'au-delà des théories que nous aimons beaucoup en France - où l'on ne s'appuie peut-être pas suffisamment sur des mesures - il faut en effet mesurer et prouver ce qu'on avance; je crois que c'est un point tout à fait capital. Quant à M. THIRRIOT et M. LEvIANDIER, ils nous ont donné une leçon d'humilité et d'espoir. Enfin, M. Roche et M. TORTEROTOT nous ont confirmé que nous pouvons effectivement garder l'espoir en la science. Maintenant, nous pouvons passer aux questions. Qui demande la parole?
\end{abstract}

M. PARSY: $\mathrm{M}^{\mathrm{me}}$ CosandeY nous a parlé de crues qui n'étaient pas de ruissellement pur, mais qui étaient en fait, des crues de nappes qu'on peut souvent expliquer ainsi : la pluie percolant rapidement augmente la charge piézométrique, la transmission de pression étant quasi-instantanée, le flot de base croît. Dans ces conditions, les temps de réponse ou de réaction sont fonction de la vitesse de percolation. Est-ce le phénomène que vous avez pu constater ?

$M^{m e}$ COSANDEY : Absolument, il ne s'agit pas de ruissellement, au sens strict du mot, et c'est pourquoi, dans mon introduction, j'ai précisé que je parlerai d' "écoulement rapide" et non de " ruissellement". L'eau qui s'écoule provient essentiellement de la nappe, par transmission rapide de pression. C'est tout à fait ce dont vous parlez.

M. PARSY: Dans ces conditions, si l'on suppose que la pluie dure suffisamment de temps pour saturer le sol, quand le niveau de la nappe atteint la surface du sol, on peut imaginer que le coefficient de ruissellement passera brutalement de 0 à pratiquement 1 . On aura un hydrogramme qui aura une forme extrêmement pointue.

$M^{m e}$ COSANDEY: Oui, avec cette réserve que le plancher imperméable n'est pas partout à la même profondeur, et donc que la nappe n'affleure pas en même temps sur toute la surface du bassin. Dans certains cas de crues cévenoles dans des petits bassins, avec des pluies très importantes, on observe une montée très rapide de l'hydrogramme à partir du moment où l'ensemble du bassin est saturé. Ce n'est évidemment pas la même chose en Bretagne, où les hauteurs de pluie sont bien moindres, et la zone saturée généralement limitée aux fonds des vallons.

M. PARSY: Cela confirme ce qu'on avait déjà vu pour Tulle.

M. DUJARDIN : Le travail de $\mathrm{M}^{\text {me }}$ CoSANDEY recoupe un travail que j'avais fait à la même époque sur des bassins bretons également.

Javais constaté que des pluies identiques pouvaient donner des crues d'une puissance variée, et trouvé l'explication dans une relation entre le ruissellement et le débit initial de la rivière, ce qui a permis de faire un outil de prévision pratique et utilisable facilement. $\mathrm{M}^{\mathrm{me}}$ COSANDEY a apporté une explication à ce phénomène qui doit en permettre et en justifier l'exploitation sur d'autres bassins.

Le Président: M. Masson nous a indiqué tout à l'heure que, dans les modèles simples, on fait intervenir l'absorption par le sol par un certain coefficient multiplicatif, c'est-à-dire que l'on considère qu'à un moment donné il y a une certaine proportion de la pluie qui est absorbée par le sol. D'autres modèles qui marchent bien par ailleurs tendent à soustraire une partie de la pluie. Je crois que le bassin que vous avez étudié, Madame, correspondrait plutôt au premier cas, ce qui veut dire que d'autres phénomènes peuvent peut-être se produire lorsque le deuxième modèle se trouve mieux adapté.

$$
M^{m e} \text { COSANDEY : Bien sûr. }
$$

M. DUBAND: Le cas des bassins bretons est très intéressant, mais il faut faire attention à l'extrapolation aux bassins cévenols parce que les intensités de précipitation sont très importantes et les nappes, dans certains cas, n'interviennent pas du tout parce que l'eau n'a pas toujours le temps de rentrer dans le sol.

$M^{m e}$ COSANDEY : Pour ma part, je pense que lorsque le sol est bien couvert par la végétation, l'infiltration des pluies est beaucoup plus importante qu'on ne l'admet généralement, même si cette eau peut réapparaître en surface un peu plus en aval, et alors ruisseler si les conditions de saturation des horizons sous-jacents sont différentes.

M. DUBAND: Il faut être prudent dans ces assertions.

Le Président : Lorsqu'on calcule le volume écoulé de la crue de la Loire dont je parlais tout à l'heure, on arrive à 20 ou $25 \%$. Donc, il y a bien de l'eau qui est partie.

M. DUBAND : Sur le volume total précipité, il est probable que, pendant quelques heures, le coefficient de ruissellement a peut-être atteint des valeurs proches de 0,8 . C'est cela qu'il faut regarder; il ne faut pas regarder seulement le coefficient global.

\section{Le Président : C'est exact.}

$M^{\text {me }}$ COSANDEY : J'ai parlé de crues sur des sols bien couverts par la végétation; je ne parle pas d'autres cas où les phénomènes qui interviennent sont différents. J'ai vu des cas de pluies simulées de $120 \mathrm{~mm} / \mathrm{h}$ qui arrivaient à des ruissellements faibles, pour ne pas dire complètement négligeables dans les Cévennes. Or ces intensités de pluies étaient supérieures à celles qui se produisent dans la réalité. Ce qui veut dire que les phénomènes qui se passent sont plus complexes, puisque le ruissellement de surface existe effectivement dans les Cévennes. Je pense que les choses se passent davantage par débordement de nappe que par simple refus à l'infiltration.

M. LeYNaUd: Lorsqu'on essaye de vérifier a posteriori la validité des modèles, on est amené à utiliser des épisodes remontant assez loin dans le temps. On présuppose dans ces opérations que la réponse du bassin versant et du lit mineur lui-même reste constante. Ne peut-on pas penser que les modifications que subissent depuis une vingtaine d'années les caractéristiques des bassins versants et des lits mineurs (je ne parle pas des ouvrages bien localisés, mais des modifications du mode d'occupation et d'exploitation des sols, des extractions de matériaux, etc.) sont suffisantes pour devoir être prises en compte, et dans quelle mesure sait-on le faire?

M. MASSON : J'ai dit qu'il fallait faire très attention à ce genre de perturbations. On doit prendre en compte les extractions de matériaux dans le lit des rivières. C'est assez compliqué, à mon avis, et ce n'est pas par une réponse de deux minutes qu'on peut faire le tour de la question. 
M. OBLED: Je voudrais faire une remarque et poser une question à $\mathrm{M}^{\mathrm{me}} \mathrm{COSANDEY}$. Voici la remarque : dans votre exposé, vous supposiez que la pluie était équirépartie sur le bassin, qu'il y avait une sorte de pluie uniforme sur le bassin. Je ne sais pas si vous pouvez nous dire comment on pourrait imaginer ce que deviendrait le processus que vous décrivez quand on a de bonnes raisons de supposer que la pluie est une sorte de juxtaposition de pluies très concentrées en certains points du bassin et beaucoup plus faibles en d'autres endroits, autrement dit, quand il y a une forte disparité spatiale de pluies. Voici la question : vous avez présenté ce concept au niveau d'un bassin d'une vingtaine d'hectares, ce qui me semble plus petit que ce dont j'avais entendu parler par ailleurs. Pouvez-vous nous donner une idée de la gamme de tailles de bassin sur laquelle vous trouvez que ce concept s'applique? Jusqu'où peut-on descendre et jusqu'où peut-on monter?

$M^{m e} \operatorname{CoSANDEY}:$ Je ne crois pas que ce soit une question de taille des bassins-versants, ce sont plutôt des facteurs climatiques, de type de sol, et surtout de couverture végétale qui interviennent. Si le sol est nu ou mal couvert, les phénomènes de surface (de type : battance) modifient complètement les conditions d'infiltration et alors c'est l'intensité des pluies, et la répartition de cette intensité dans le temps et dans l'espace qui joue le rôle essentiel.

Mais, sous nos climats, si le sol est bien couvert par la végétation, je pense que c'est de beaucoup l'infiltration qui domine et que, lorsqu'il y a ruissellement, c'est plus par débordement de nappe que par refus d'infiltration d'un sol non saturé. Ce type de ruissellement (combiné à d'autres parce que le sol n'est pas partout protégé par la végétation) se produit sur les bassins versants du Mont-Lozère, où l'épaisseur du sol est de l'ordre de $50 \mathrm{~cm}$ et où des pluies atteignant 50 et $60 \mathrm{~mm}$ en une heure peuvent s'observer.

$\mathrm{Si}$ ces processus ne dépendent guère de la taille des bassinsversants, par contre la complexité des combinaisons de processus provoquant le ruissellement augmente forcément avec la taille du bassin.

M. OBLED : Vous pensez qu'il y a un phénomène généralisé ?

$M^{m e} \operatorname{Cos} A N D E Y$ : Oui, quand les sols ne sont pas nus.

M. ERLICH : Je voudrais poser une question concernant la dernière présentation d'exposé de MM. ROCHE et TORTEROTOT. Vous parlez des décisions en temps réel sur la base des modèles déjà établis. Or, on sait très bien que l'incertitude concerne aussi le réglage des modèles. Par exemple, les performances correctives du filtre de Kalman dépendent des conditions initiales; il y a donc une décision à prendre aussi à ce niveau.

M. ToRterotot: Pour les exemples que j’ai présentés, nous avons utilisé des modèles linéaires et uniquement des relations entre débits ou entre variations de débits, en respectant autant que faire se peut les règles d'usage en matière de calage : des crues homogènes qui soient représentatives de ce qui se passe, avec un nombre de données suffisant.

Concernant le filtrage, nous le destinons à prendre en compte l'évolution à moyen et long termes des conditions d'écoulement des crues du bassin. On ne lui demande donc pas de réagir de façon sensible à chaque pas de temps; les matrices de covariance peuvent être réglées de manière à ce que la réponse du filtre soit assez lente. En mise en œuvre opérationnelle, il faudra s'assurer d'une durée de mise en route suffisante du filtre, pour que ce dernier puisse recaler les paramètres du modèle avant la phase de montée rapide de crue.

M. P.A. ROCHE : Je voudrais donner une information complémentaire concernant le filtrage. La procédure que nous utilisons consiste à explorer les matrices de variance d'observation et d'évolution en prenant une règle qui est un peu issue de l'expérience : l'essentiel de l'exploration se fait le long du rapport des traces des deux matrices et, en fait, tout le reste est assez négligeable par rapport à ce premier facteur qu'on appelle facteur de sensibilité du filtre; on règle la sensibilité du filtre et son aptitude à réagir rapidement en fonction de ce qu'on veut lui faire faire. Dans l'expérience qui a été faite, on lui a demandé de réagir rapidement; mais, pour un usage opérationnel destiné à la correction des évolutions à long terme, il va falloir brider sa sensibilité pour le faire réagir beaucoup plus lentement. Comme nous l'avons dit, il ne faut pas concevoir le filtrage comme un substitut au traitement des écarts importants à court terme, mais comme un moyen de recalage des paramètres du modèle à long terme. Le deuxième point concerne le problème que vous citez d'initialisation de la covariance de l'état du système, c'est-à-dire des paramètres du modèle. La méthode qui est utilisée est un peu empirique; on part d'une estimation au début de chaque crue. Cette estimation de la matrice de covariance est faite à l'aide d'un certain nombre d'itérations de l'algorithme de Kalman (par exemple 100 itérations), en prenant les données des premiers pas de temps. On obtient ainsi une matrice de covariance qui est cohérente avec cette information. Il est certain que si l'on ne fait pas cela et si l'on ne cale pas cette covariance en début de chaque crue, on peut obtenir des gains de filtre qui sont très largement surestimés par rapport à la confiance à accorder aux paramètres. Au contraire, si l'on a sous-estimé la confiance à accorder aux paramètres du modèle en prenant une initialisation artificiellement trop forte pour cette matrice de covariance des paramètres, ils ne vont pas réagir sur les pas de temps qui nous intéressent qui sont au début de la crue. Ils ne vont donc pas répondre à ce que l'on souhaite.

M. LEVIANDIER: Je ne suis pas tout à fait d'accord sur le fait que, d'une façon générale, le filtrage soit réservé aux seules actions à long terme. Dans le modèle un peu plus conceptuel sur lequel j'ai travaillé, on peut faire un filtrage différentiel : le niveau du réservoir sol étant filtré assez faiblement parce qu'il varie à long terme, et le niveau du réservoir hydraulique peut assurer par un filtrage plus énergique, la correction à court terme.

M. THIRRIOT: Je voudrais poser une question à M. ROCHE et à vous-même. Vous avez dit à plusieurs reprises qu'en définitive ARIMA et filtre de Kalman revenaient à peu près à la même chose. Il me semble que dans le modèle ARIMA, on va chercher quelque chose qu'il n'y avait pas encore dans le système étudié, et dans les filtres de Kalman on va introduire quelque chose qui est un peu comme une non linéarité. Je voudrais avoir votre sentiment ou votre expérience sur cette question de clivage entre l'aspect innovation des modèles ARIMA et l'aspect filtre de Kalman qui va, en somme, incorporer une prise en compte de non linéarité.

M. P.A. ROCHE : L'intérêt que nous avons porté au couplage du filtrage et des traitements d'écarts est issu du constat que nous avons fait que, dans de nombreux cas, nous ne faisions par le filtrage qu'une réévaluation d'un décalage : cette forme très simple de filtrage devait bien avoir un équivalent dans la modélisation des processus d'écarts.

Corriger, ainsi que vous le proposez, à court terme un paramètre du modèle me semble surtout traduire une imperfection de la modélisation. Plutôt que de croire que l'on a réadapté un paramètre, que l'on va remettre en cause entièrement dès le pas de temps suivant, il vaut mieux accepter que le processus des écarts du modèle aux observations est susceptible de comporter des persistances, et être non stationnaire.

Pourquoi un processus ARIMA ? Nous étions partis sur l'idée que l'équivalent du filtrage d'une constante était un ARI. C'est en faisant le calcul, et afin d'assurer la cohérence entre les deux approches, que nous avons vu que la structure de filtrage introduisait la moyenne mobile et pas seulement l'aspect non stationnaire. En y réfléchissant, c'est d'ailleurs tout naturel : le filtrage n'est qu'un opérateur de lissage, dont l'expression la plus simple est la moyenne mobile.

$M$. GRARD : Je reviens un peu en arrière, sur la communication de $\mathbf{M}^{\text {me }}$ COSANDEY, concernant l'utilisation de l'échantillon.

J'ai été heureux de constater que, pour la seule fois de la journée, on est bien parti de la cause vers l'effet et non l'inverse, en, ce sens que $\mathbf{M}^{\text {me }}$ COSANDEY a choisi son échantillon à partir des pluies et non utilisé un échantillon de crues pour déterminer l'effet des pluies. Je pense que c'est assez capital si l'on veut vraiment, en prévision à la suite d'averses fortes, avoir des possibilités de non-crue, car la non-crue est un résultat de prévision non négligeable si l'on veut éviter des alertes inutiles.

M. BocQuillon: Je reviens sur la communication de $\mathbf{M}^{\mathrm{mc}}$ COSANDEY. Il me semble que c'est une notion difficilement généralisable pour une excellente raison : si l'on admet qu'il y a des surfaces ruisselant à $100 \%$, ces surfaces sont localisées et toutes petites vis-à-vis de la totalité du bassin. Aucun modèle pluie-débit ne pourrait fonctionner avec cette hétérogénéité. Je 
pense que c'est une notion sur laquelle il faut se poser des questions. D'autres explications ont été données qui me paraissent beaucoup plus rationnelles, et sont relatives à la transmission par la nappe.

$M^{m e}$ COSANDEY : L'idée selon laquelle seule une petite partie du bassin-versant contribue au ruissellement de surface n'est plus nouvelle. Probablement la variation de l'extension de ces «surfaces ruisselantes " dépend en partie de transmission de pression dans la nappe. Pour ce qui est du transfert rapide de l'eau entre son point de chute et l'exutoire (et qui constitue la crue), les processus de ruissellement de surface - stricto sensu - et de transmission de pression doivent intervenir respectivement pour des parts extrêmement variables suivant les événements. Sur les versants bien couverts par la végétation, l'essentiel de la circulation de l'eau se fait probablement davantage par écoulement latéral dans l'épaisseur du sol, au-dessus du niveau imperméable ( " hypodermisme " au sens de TRICART) que par ruissellement sur les versants, du moins tant que ceux-ci ne sont pas saturés.

M. BocQuillon: Je pense qu'il aurait été plus rationnel d'utiliser un modèle avec plusieurs zones de pluie afin de voir l'influence de cette spatialisation de la pluie sur la réponse.

$M^{m e}$ COSANDEY: Dans un petit bassin en milieu forestier, l'hétérogénéité des mesures de précipitation est bien supérieure à l'hétérogénéité des phénomènes qui surviennent en milieu océanique. Je ne pouvais pas aller plus loin que ce que jai fait dans cette voie.

M. BOCQUILLON: On pourrait généraliser à une grande surface.

\section{$M^{m e}$ COSANDEY : C'est une possibilité.}

Le Président: Je voudrais reprendre ce qui a été dit tout à l'heure à propos de votre travail. Il y a un point sur lequel je voudrais revenir et qui me paraît fort important : il s'agit de la corrélation entre le débit de base avant la crue et le ruissellement tel qu'on peut le prévoir pour la première pluie.

Jai le souvenir que dans le bassin de la Corrèze à Brive, dont j’ai parlé, le $\Delta t$ était de $4 \mathrm{~h}$. On mesure donc les pluies en $\mathrm{mm}$ par $4 \mathrm{~h}$. La corrélation entre le débit de base et l'absorption par le sol dans les 4 premières heures est tout à fait satisfaisante; elle permet de prévoir les "non crues" et cette absorption varie de quelques mm lorsque le sol est saturé. l'hiver, jusqu'à 40 à $50 \mathrm{~mm}$. La plage est donc énorme. Le fait que l'on sache avec une bonne probabilité qu'il va se produire des pluies importantes ou qu'il ne va rien se passer du tout (prévisions météo) est tout à fait essentiel et les bonnes corrélations absorption-débit de base sont alors très utiles.

M. PARSY: Je ne suis pas tout à fait daccord avec $\mathbf{M}$. BOCQUILLON, l'expérience ayant montré que, pour les $360 \mathrm{~km}^{2} \mathrm{du}$ bassin de Tulle, tout se passait, tant qu'on ne dépassait pas une centaine de $\mathrm{m}^{3} / \mathrm{sec}$, comme si l'on avait une crue de nappe. Ce n'est que lorsqu'on dépassait $100 \mathrm{ou} 110 \mathrm{~m}^{3} / \mathrm{s}$ qu'on avait des crues très pointues. La crue de 1960 , avec $80 \mathrm{~mm}$ faisant suite à des pluies relativement longues en temps: à 15 jours, qui avaient saturé les sols, a donné une pointe de crue aux environs de 400 $\mathrm{m}^{3} / \mathrm{s}$, alors qu'une pluie tombant sur ce même bassin, de $120 \mathrm{~mm}$ en moins de $24 \mathrm{~h}$, n'a strictement rien donné et qu'on a pu mesurer des taux d'infiltration allant jusqu'à $1000 \mathrm{~mm} / \mathrm{h}$.

De plus, le sol n'est pas homogène sur les $360 \mathrm{~km}^{2}$ parce qu'on a également pas mal d'arênes granitiques, et je crois que le résultat moyen était quand même une crue de nappe pour les crues moyennes. Je vous rejoins tout à fait pour dire : il ne faut pas généraliser ni dans un sens ni dans lautre en confirmant la nécessité de prendre en compte l'hydrogéologie.

M. DUBAND: Depuis une vingtaine d'années, on a trouvé les indicateurs de saturation d'un bassin dans le Massif Central, dans le Morvan, dans le Jura, mais pas du type Cévenol, c'est-à-dire un haut bassin. On s'aperçoit qu'il y a des épisodes qui présaturent lentement l'indicateur, mais il y a des réactions avec un débit de base pratiquement nul avec des intensités de 60 à $90 \mathrm{~mm} / \mathrm{h}$. Il faut donc être très prudent.

$M^{m e}$ COSANDEY : Je suis bien entendu d'accord sur la prudence ! Rien n'est jamais si simple dans les milieux naturels... Ceci dit, avec des précipitations de 60 ou $80 \mathrm{~mm}$ en une heure, il est possible dans certaines régions cévenoles, étant donné la faible épaisseur du manteau détritique, d'avoir des débordements de nappe importants.

M. DUBAND: C'est différent s'il n'a pas plu dans les 15 jours ou 30 jours qui ont précédé.

$M^{m e}$ COSANDEY : Cela dépend de l'état du sol, et notamment du prélèvement d'eau effectué par l'évapotranspiration. Ce qui serait très intéressant - et j'espère que cela va pouvoir se réaliser - c'est de faire des analyses isotopiques qui permettraient de déterminer avec précision l'origine de l'eau qui forme l'écoulement de crue.

M. SotTY: Jaimerais insister sur une réserve que faisait $\mathbf{M}^{\text {me }}$ COSANDEY sur la nature des sols, où elle faisait apparaître le problème de l'état de surface et de la couverture végétale.

On note fréquemment, dans le Sud-Est notamment, des sols qui, en vallée, sont fortement argileux, imperméables, et sur le relief, beaucoup plus calcaires et beaucoup plus perméables. II semblerait que les essais faits avec un simulateur de pluie mettent en évidence que l'on arrive à un phénomène de battance très facile à voir; pour une pluie inférieure à une certaine intensité, on a généralement une percolation. Par contre, à partir d'une intensité critique, on voit brusquement le ruissellement qui se produit. Jaimerais poser la question à $\mathbf{M}^{\text {me }}$ COSANDEY et à $M$. DUBAND : est-ce que la réponse n'est pas entre les deux : pour des pluies d'importance faible, on aurait mise en fonctionnement de la nappe, et par contre, pour des intensités plus importantes, notamment à partir des fréquences quinquennales ou décennales, on aurait un phénomène de ruissellement en grand?

M. THIRRIOT: M. SotTy pourrait-il préciser si les fortes intensités dont il parle dépassent la perméabilité à l'absorption du sol ?

M. SotтY: J'ai à l'esprit des travaux qui ont été faits dans la région de Nice notamment par le groupe du BRGM de Marseille. Personnellement, je suis très prudent et très sceptique sur la possibilité d'extrapoler les observations faites sur de petites surfaces aux bassins importants. Par contre, en travaillant sur des terrains karstiques, on a l'impression que le phénomène de saturation est exprimé non pas en termes de débit initial, mais plutôt en termes d'intensité de la pluie.

$M^{m e}$ COSANDEY : Les processus sont complètement différents à partir du moment oủ le sol n'est plus couvert de végétation, alors interviennent des phénomènes qui changent complètement les conditions de formation du ruissellement. Dans ce cas, il semble que le facteur essentiel soit l'intensité de la pluie. Si la pluie commence par une intensité très forte et diminue ensuite, le sol sera tassé par la première pluie et se mettra à ruisseler. Les phénomènes sont complètement différents sur sol nu et sur sol bien couvert par la végétation. Encore une fois, je ne veux pas être trop catégorique. Dans les cas où le sol est bien couvert, il peut absorber même une précipitation cévenole. Il y a un ruissellement important, mais qui se fait, à mon avis, par d'autres processus que de simples processus de refus d'infiltration du sol de type Horton.

M. DUJARDIN : Je voudrais poser deux questions à M. TORTEROTOT. Sur la modélisation des erreurs d'un modèle et la correction des écarts en cours de crues, je pose la question suivante : est-ce qu'il vous paraît possible et prudent d'intervenir à ce moment-là, non pas sur la modélisation des écarts, mais sur les paramètres du modèle conceptuel initial, pour la prévision suivante? A mon avis, c'est relativement dangereux parce qu'il peut $\mathrm{y}$ avoir une erreur de mesure, qui nous conduirait à une correction non justifiée. Ma deuxième question concerne la mise en cuvre en parallèle de deux méthodes de prévision pour regarder ensuite laquelle des deux il faut choisir. Cela me parait difficile parce que je conçois mal que l'on puisse avoir deux modèles ayant les mêmes objectifs mais utilisant des données différentes. Par contre, on peut avoir un modèle pour une prévision à court terme et un autre pour un délai plus long, utilisant des stations entrée plus en amont. Mais comment les comparer et les valider l'un par rapport à l'autre ? Est-ce que la procédure n'exige pas une autre précaution qui consisterait à avoir une redondance de mesures permettant de les sélectionner et de les corriger avant d'entrer dans les modèles et non pas à l'examen de leurs résultats ?

M. TORTEROTOT: Je vous répondrai d'abord sur la deuxième question. Toute sécurité apportée à l'amont de la phase de 
prévision est précieuse. Mais la redondance de la mesure ne peut apporter de réponse qu'aux erreurs de mesure, dans le cas où on sait distinguer la valeur juste de la fausse.

Si l'on veut faire une critique sur une donnée en se servant des cohérences existant entre différents indicateurs, on passe nécessairement par l'intermédiaire de modèles, et donc on apporte une correction à l'aval de la phase de prévision.

Par ailleurs, considérer des modèles à des échéances de prévision différentes revient à faire des prévisions pour un même pas de temps à partir d'indicateurs différents. Même, si en cas de perturbation, on ne sait pas trancher entre deux modèles, la procédure de combinaison permettra de stabiliser la qualité de la prévision globale.

Pour répondre à la première question, le filtre sera calé pour ne pas être trop entreprenant. Son rôle est de faire évoluer les paramètres à moyen terme. Une perturbation ponctuelle aura donc un impact faible sur ces paramètres.

M. THIRRIOT: M. DUjaRdin a suggéré une question qui pourrait conduire à un très long débat : il s'agit de la redondance. Que pensez-vous du traitement de la redondance? Pensez-vous qu'actuellement, dans les modèles utilisés, même s'ils sont simples, l'on gère correctement le risque de redondance et le risque d'instabilité ? Je pose la question à toute la salle ?

M. P.A. RoCHE: Je crains que, dans ce débat, nous nous écartions un peu des réalités, et je ne voudrais pas que l'on s'engage sur des discussions un peu trop byzantines, sur des choses qui, finalement, n'ont pas encore bénéficié d'une expérience opérationnelle et en sont à leurs premiers essais. Lorsqu'on teste simplement un modèle donné et qu'on ajoute un traitement d'écarts, l'expérience montre que c'est quelque chose, qui d'une part, est peu ou pas utilisé par les prévisionnistes et, d'autre part, est très dangereux. Il $\mathrm{y}$ a eu plusieurs études oủ des gens, en voulant faire du traitement d'écarts et réduire la dispersion moyenne, ont créé des oscillations notamment au voisinage des crues. On trouve dans les études des oscillations qui arrivent même à des ruptures de l'hydrogramme. De telles procédures n'ont guère de chance d'être utilisées par les prévisionnistes. C'est d'ailleurs facile à comprendre. Comme les méthodes de base dont parlait M. THIRRIOT et qui consistent à extrapoler dans le temps les données de la station aval, traiter les écarts d'un modèle en une station c'est être totalement myope vis-à-vis de l'ensemble du bassin. Arriver à une vision plus globale du bassin est le but que nous recherchons : il n'y a qu'à ce niveau que l'on peut trouver des méthodes assez stables pour respecter la structure des hydrogrammes, ce qui me semble une nécessité absolue pour l'application.

Lorsque vous dites que les stations amont correspondent à une propagation plus importante et ne répondent pas à l'échéance de prévision, c'est certain, mais cela n'empêche pas pour autant d'utiliser ces indicateurs pour une échéance plus courte. Il suffit d'aller chercher des données un peu plus tôt, et c'est ce que nous faisons en fait. $\mathrm{Si}$, par exemple, j'ai un doute sur la donnée de Larche (bassin de la Vézère), il faut bien que je fasse quelque chose en remplacement, et ce quelque chose, c'est en fait un substitut à l'amont. Je préfère utiliser directement l'indicateur amont et avoir fait le travail à l'avance plutôt que de le faire en cours de crue.

Comment gérer la redondance entre les stations ? M. VIDAL a déjà signalé le volume de l'information que les services d'annonce de crue doivent interpréter chaque jour. Prenons le cas de deux capteurs soumis aux problèmes d'erreurs de transmission. Il faut faire une interprétation sur la forme, la cohérence, etc. M. PARSY nous a montré ce travail. Bien souvent, il va falloir non pas deux capteurs à distance, mais en ajouter un troisième. Si l'on voulait vraiment acquérir une information totalement fiable, il y aurait, au niveau des services opérationnels, des difficultés absolument invraisemblables. Il n'est pas question de s'engager dans cette voie, compte-tenu du coût et des problèmes de main-d'œuvre, et c'est dommage. On est amené à rechercher une assez grande fiabilité des méthodes de prévision vis-à-vis des perturbations locales. Si l'on veut vraiment gagner quelque chose il faut aller chercher de nouvelles informations. C'est, en fait, ce que fait le prévisionniste. 